\title{
Appraisal of electromagnetic induction effects on magnetic pulsation studies
}

\author{
B. R. Arora ${ }^{1}$, P. B. V. Subba Rao ${ }^{1}$, N. B. Trivedi ${ }^{2}$, A. L. Padilha ${ }^{3}$, and I. Vitorello ${ }^{3}$ \\ ${ }^{1}$ Indian Institute of Geomagnetism, Colaba, Mumbai 400 005, India \\ ${ }^{2}$ Southern Regional Center for Space Research - CRSPE LACESM/CT/UFSM, Santa Maria, RS 97119-900 Brazil \\ ${ }^{3}$ Instituto Nacional de Pesquisas Espaciais, Sao Jose dos Campos, SP 12201-970, Brazil
}

Received: 12 July 2000 - Revised: 25 October 2000 - Accepted: 28 November 2000

\begin{abstract}
The quantification of wave polarization characteristics of ULF waves from the geomagnetic field variations is done under 'a priori' assumption that fields of internal induced currents are in-phase with the external inducing fields. Such approximation is invalidated in the regions marked by large lateral conductivity variations that perturb the flow pattern of induced currents. The amplitude and phase changes that these perturbations produce, in the resultant fields at the Earth's surface, make determination of polarization and phase of the oscillating external signals problematic. In this paper, with the help of a classical Pc5 magnetic pulsation event of 24 March 1991, recorded by dense network of magnetometers in the equatorial belt of Brazil, we document the nature and extent of the possible influence of anomalous induction effects in the wave polarization of ULF waves. The presence of anomalous induction effects at selected sites lead to an over estimation of the equatorial enhancement at pulsation period and also suggest changes in the azimuth of ULF waves as they propagate through the equatorial electrojet. Through numerical calculations, it is shown that anomalous horizontal fields, that result from induction in the lateral conductivity distribution in the study region, vary in magnitude and phase with the polarization of external source field. Essentially, the induction response is also a function of the period of external inducing source field. It is further shown that when anomalous induction fields corresponding to the magnitude and polarization of the 24 March 1991 pulsation event are eliminated from observed fields, corrected amplitude in the $X$ and $Y$ horizontal components allows for true characterisation of ULF wave parameters.
\end{abstract}

Key words. Geomagnetism and paleomagnetism (geomagnetic induction) - Ionosphere (equatorial ionosphere) - Magnetospheric physics (magnetosphere-ionosphere interactions)

\section{Introduction}

Magnetic pulsations as recorded by a network of magnetometers are useful tools to investigate the complex magne-

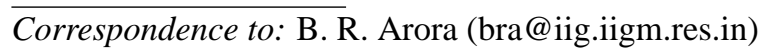

tospheric processes, in general, and to understand the generation and propagation mechanisms of ULF waves, in particular (Hughes, 1994). In such studies, determination of wave polarization parameters (ellipticity, degree and sense of polarization, azimuth and phase etc.) from the recorded geomagnetic variations, inevitably form the first step. In all frequency ranges, the geomagnetic field variations are the vector sum of the contributions from the external (source) current system in the ionosphere/magnetosphere and their counterparts, resulting from the electromagnetic induction in the electrically conductive layers of the Earth. In the absence of lateral variations in internal conductivity, the internal currents are simply the mirror images of the external source current systems and, as such, their magnetic effects in the horizontal $(X$ and $Y$ ) field components are in-phase with the inducing source fields. Only in such idealized cases can the polarization and phase properties of the oscillating external signals be determined uniquely from the recorded total fields (Egbert and Booker, 1993). Over such an Earth model, where conductivity varies only as a function of depth, the observed fields, which are the sum of external plus the induced part in the stratified Earth, are referred to as normal field components (Schmucker, 1970). Lateral conductivity variations, either in the form of land-ocean contrast or those associated with crustal and mantle structures, perturb the flow pattern of induced currents, producing local anomalies in the magnetovariational field components. The isolation and interpretation of the frequency dependent anomalous fields are the subject matter of Geomagnetic Deep Sounding (GDS) that is aimed at mapping the conductivity structures of the Earth's crust and upper mantle (Gough and Ingham, 1983; Arora, 1997). The depth control comes from the well-known skin-depth relationship; depth penetration increases with the increasing period of oscillating external fields. On the other front, the amplitude and phase changes that these perturbations produce in the total observed fields make the precise determination of the external source characteristics problematic. In this paper, we present evidence that anomalous induction effects can bias the quantification of the external source characteristics. This is documented with the help of an illustrative example of Pc5 magnetic pulsations, as recorded by a dense 


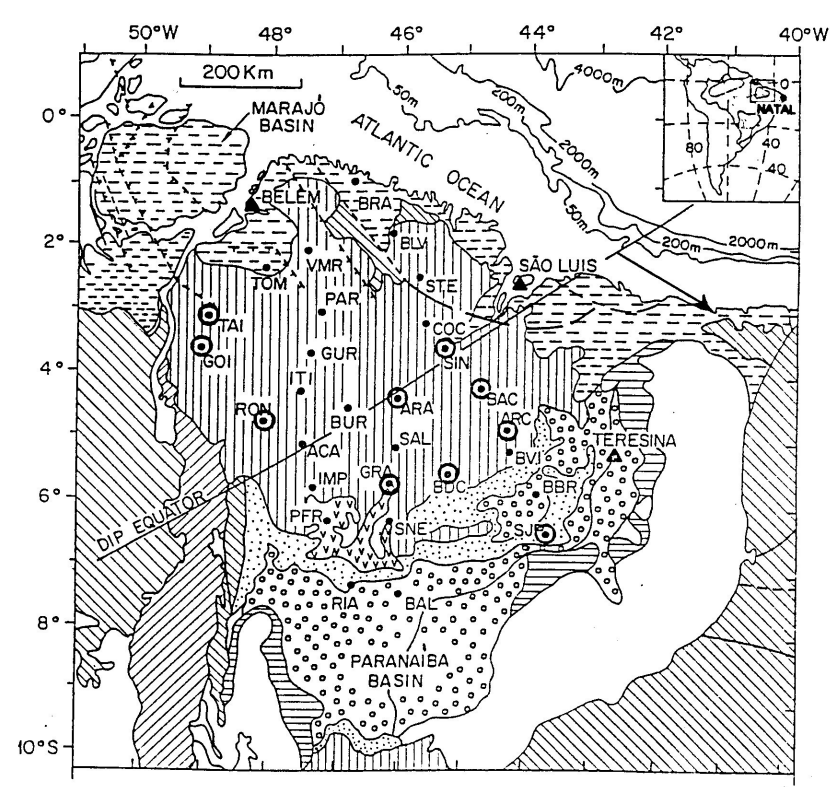

Fig. 1. Map of N-NE Brazil showing the location of magnetometer sites in relation to the dip equator. Stations that recorded the Pc5 Pulsations associated with magnetic storm of 24 March 1991 are circled. Inset shows the location of study area on the map of South America. Location of station Natal, in relation to the array area, is also marked in the inset. Exact location of Natal is positioned at a distance of four times the length of the arrow, along the direction of arrow.

array of magnetometers in the equatorial region of the N-NE Brazil. Given this observational evidence, useful guidelines to estimate the extent of induction effects are briefly outlined.

\section{Pc5 magnetic pulsations of 24 March 1991}

Figure 1 shows the layout of a 29-station magnetometer array that was operated in the equatorial region of the northnortheast Brazil between November 1990 and March 1991. The primarily aim of this survey was to map the internal conductivity distribution of the large Parnaiba basin through the principles of GDS (Arora et al., 1997; 1998). The electrical conductivity distribution in and around the array area, as deduced by the thin sheet modeling of the anomalous induction response, is shown in Fig. 2. From the nature of conductance distribution, two major conductive structures were inferred (Arora et al., 1997):

1. Parnaiba Basin Conductivity Anomaly (PBCA) - Positioned close to the central part of the Parnaiba basin; it extends NE-SW along the southeastern part of the Parnaiba basin. This anomaly is also found to enclose a localized resistive zone.

2. The second LINK Conductivity Anomaly connects the Parnaiba and Marajo basins. The anomaly branching off from the central part of the PBCA extends towards the Marajo basin in a NW-SE direction.

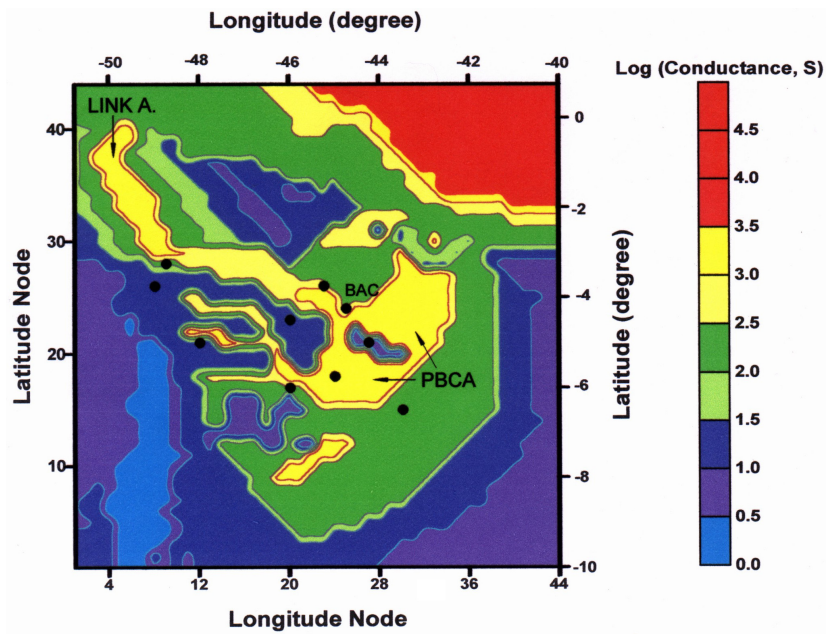

Fig. 2. Nature of electrical conductance distribution in the N-NE Brazil, as deduced by the thin-sheet modelling of anomalous induction effects (Arora et al., 1997). PBCA (Parniaba Basin Conductivity Anomaly) and LINK anomalies are zones of high conductance. Locations of stations that recorded Pc5 event are also indicated.

A large part of the study area falls under the influence of the intense band of the Equatorial Electrojet (EEJ) current that, centered over the dip-equator, flows eastward during daytime in the ionospheric E-region. Towards the closing phase of the array operation, a sequence of Pc5 pulsations was recorded in association with the magnetic storm of 24 March 1991. Occurrence of this event of Pc5 pulsation has been reported at worldwide observatories (Fujitani el al., 1994; Trivedi et al., 1997a). The globally coherent event was recorded at ten of the array stations (see Fig. 1 for locations) as well as at station Natal, just outside the influence of the EEJ. One minute values of geomagnetic components were filtered using a zero phase shift Butterworth function with unit response in the period range of 3-15 min. The filtered field variations in the $Y$ (East), $X$ (North) and $Z$ (Vertical) field components for the period of 1100-1400 UT are shown in Fig. 3. A well developed train of Pc5 pulsations is seen at all stations, centered at 1300 UT. Spectral analysis have shown a pulsation period close to $10 \mathrm{~min}$. Polarization parameters, obtained using standard procedure described in Kanasewich (1981), are given in Table 1 and the resulting polarization ellipses, in horizontal plane, are shown in Fig. 4. One imminent feature seen in Fig. 3 is that the amplitude of the train of pulsation in the north-south $(X)$ component is considerably enhanced at stations in the immediate vicinity of the dip equator. Given this dominance of the $X$ component, the polarization ellipses are oriented in the N-S direction.

Combining the data from the present chain of stations (only those free from anomalous induction effects - documented later) with other mid- and high-latitude stations along American sector, Trivedi et al. (1997b) examined the amplitude and polarization parameters of this globally coherent Pc5 event right from the auroral latitude to the equatorial latitudes. The 


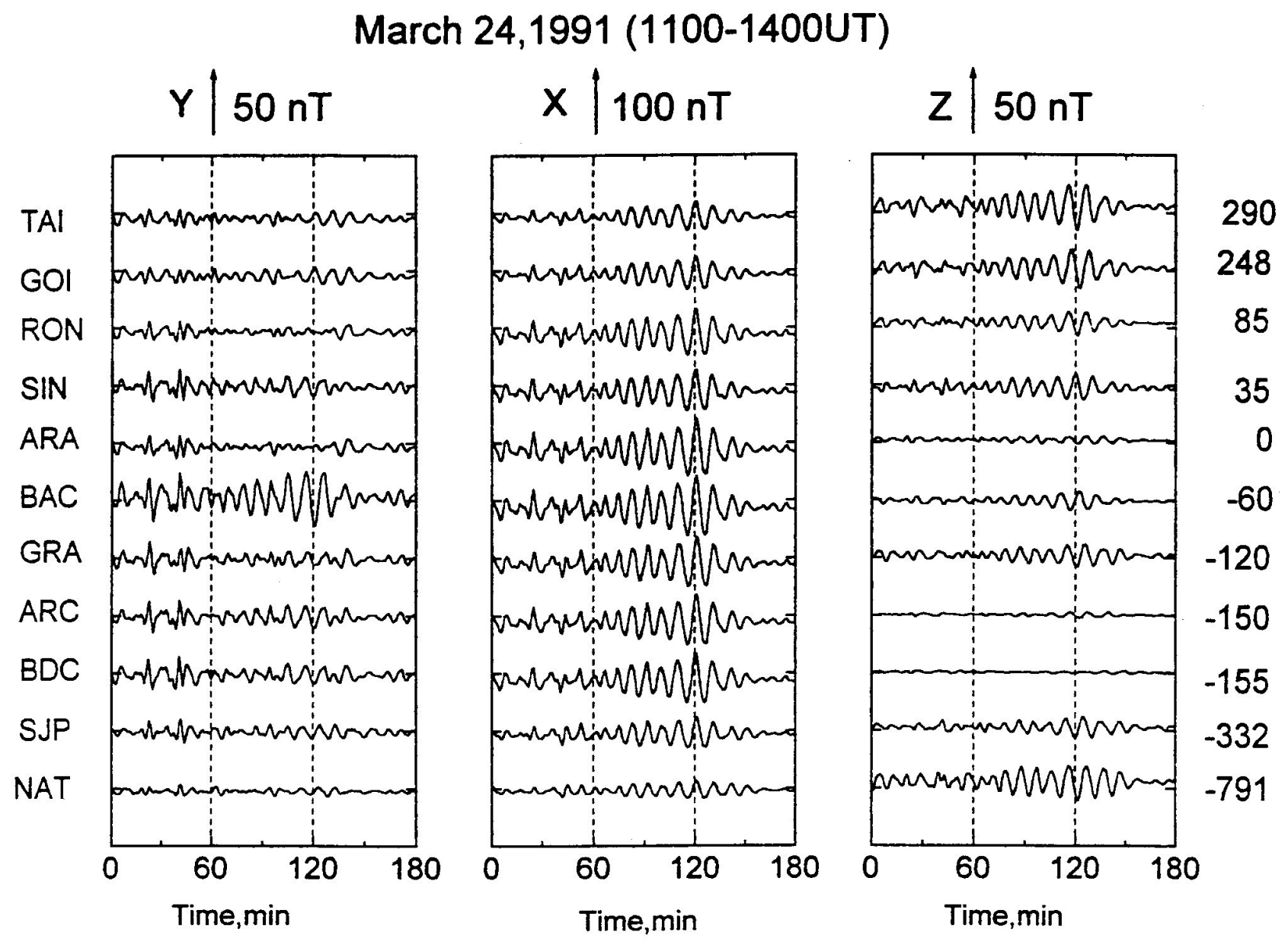

Fig. 3. Filtered geomagnetic field variation in $Y, X, Z$ in association with the Pc5 pulsations during 1100-1400 UT, 24 March 1991. Plots are stacked according to the distance of stations with respect to the dip equator.

Table 1. Polarization parameters for the Pc5 event of 24 March 1991 (1100-1400UT)

\begin{tabular}{|c|c|c|c|c|c|c|c|}
\hline \multirow{2}{*}{$\begin{array}{l}\text { Station } \\
\text { Code }\end{array}$} & \multicolumn{2}{|c|}{ Geographic } & \multirow{2}{*}{$\begin{array}{c}\text { DIP } \\
\text { (Deg.) }\end{array}$} & \multirow{2}{*}{$\begin{array}{c}\text { PSP } \\
(\mathrm{nT} 2)\end{array}$} & \multirow{2}{*}{$\begin{array}{c}\text { DEGP } \\
\text { (Percent) }\end{array}$} & \multirow{2}{*}{$\begin{array}{c}\text { AZI } \\
\text { (Deg.) }\end{array}$} & \multirow[t]{2}{*}{ ELP } \\
\hline & $\begin{array}{l}\text { Latitude } \\
\text { (Deg. S) }\end{array}$ & $\begin{array}{c}\text { Longitude } \\
\text { (Deg. W) }\end{array}$ & & & & & \\
\hline TAI & 3.15 & 48.97 & 7.9 & 9 & 96 & 11 & 0.07 \\
\hline GOI & 3.68 & 49.08 & 4.2 & 11 & 96 & 14 & -0.02 \\
\hline RON & 4.82 & 48.15 & 1.2 & 20 & 97 & 0 & -0.04 \\
\hline SIN & 3.60 & 45.39 & 0.2 & 17 & 97 & -11 & 0.04 \\
\hline ARA & 4.42 & 46.09 & -0.3 & 30 & 98 & 3 & -0.02 \\
\hline BAC & 4.24 & 44.80 & -1.6 & 40 & 99 & -23 & -0.02 \\
\hline GRA & 5.77 & 46.20 & -2.6 & 24 & 97 & -5 & -0.04 \\
\hline ARC & 4.93 & 44.38 & -3.3 & 24 & 98 & -11 & 0.02 \\
\hline $\mathrm{BDC}$ & 5.56 & 45.27 & -3.3 & 24 & 96 & -8 & -0.03 \\
\hline SJP & 6.48 & 43.76 & -6.6 & 10 & 97 & -3 & 0.19 \\
\hline NAT & 5.83 & 35.20 & -15.6 & 4 & 97 & 3 & 0.15 \\
\hline PSP & Pola & spectı & ower & & & & \\
\hline DEGP & DegI & of polariz & & & & & \\
\hline AZI & - Azin & h w.r.t. No & & & & & \\
\hline ELP & - Ellip & & & & & & \\
\hline
\end{tabular}




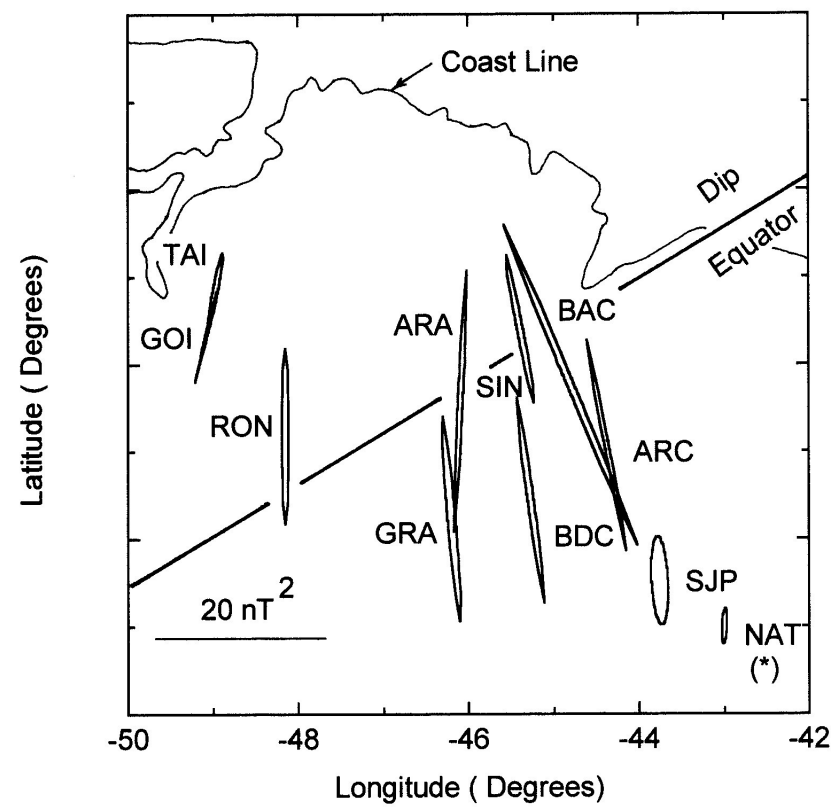

Fig. 4. Polarization ellipses for the Pc5 event of 24 March 1991 (1100-1400 UT). Length of major axis represents magnitude of polarized spectral power. Location of NAT is arbitrarily marked.

latitude-Polarized Spectral Power (PSP) plot, shown in Fig. 5 , indicates that, apart from the expected sharp maximum near the auroral oval, the pulsation amplitudes are considerably enhanced in the equatorial belt. The enhancement of the pulsation amplitude at dip equator, in comparison with lowlatitudes, clearly emphasize the role of enhanced Cowling conductivity in the equatorial ionosphere. Such visualization is well supported by the nature of equatorial enhancement that falls off to low values within \pm 4 dip latitude. Trivedi et al. (1997b) found that polarization characteristics of the pulsations across the auroral oval were consistent with the field line resonance model (Southwood, 1974). The occurrence of Pc5 pulsations at low and equatorial latitudes was attributed to the instantaneous transmission of the polar electric field, set up by field line resonance, to the low and equatorial latitudes through the ionosphere. Such hypothesis is consistent with the numerical calculations by Tsunomura and Araki (1984). They showed that the amplitude of the polar electric fields would decrease as it propagates towards the equator, but would still cause equatorial enhancement due to the increased Cowling conductivity. There is of considerable interest to quantify the spatial-frequency characteristics of this equatorial enhancement, as they are the diagnostic of the relative roles of Hall and Pederson conductivities.

\section{Physical appraisal of induction effects}

An additional feature of Fig. 3 is that the field variations in the east-west $(Y)$ component at station BAC are considerably large, although other stations with similar locations in relation to the dip equator do not show this anomalous behaviour.

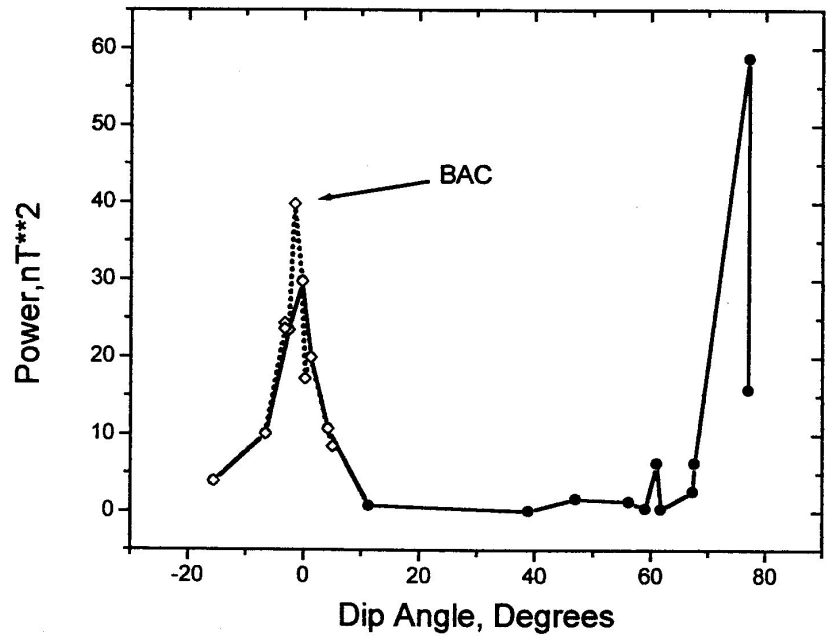

Fig. 5. Variation of Polarized Spectral Power (PSP) as a function of dip latitude - solid curve (Trivedi et al., 1997b). Broken curve shows the variation in PSP within the equatorial region when data from stations like BAC, contaminated by the anomalous induction effects, are included.

The nature of spatial conductivity distribution in the Parnaiba basin shows that the station BAC is located near the junction of two major conductive structures, namely the PBCA and LINK conductivity anomalies. 2-D modelling of induction response functions helped to visualize the PBCA as a graben structure at the base of the basin, filled with conductive carbonaceous carbonates (Arora et al., 1999). A resistive zone, found enclosed in the PBCA, is seen as intruded dibase-dikes in association with the Jurassic-Cretaceous magmatic activity. It was noted by Arora et al. (1998) that the presence of a resistive body embedded in the PBCA causes deflection and chanelling of induced currents beneath BAC. This internal induction effects account for the anomalous $Y$ fields at BAC. A net consequence of the enhanced $Y$ fields is that the polarization ellipse at BAC is considerably enlarged and its azimuth rotated in relation to the adjoining stations. When the polarized power at BAC and some other stations are included in the latitudinal profile (Fig. 5), the anomalously large power at BAC leads to a highly exaggerated estimate of the equatorial enhancement. The anomalous feature seen may also lead to inference that the enhanced ionospheric conductivity associated with the EEJ causes rotation in the azimuth of the polarization ellipse. If the estimate of the equatorial enhancement were based on a single equatorial station, as often is the case, the effect of induction bias would largely remain unknown. This anomalous enhancement factor, if not guarded for induction bias, would over emphasize the role of Hall conductivity in relation to the Pedersen conductivity in producing the equatorial enhancement, and place serious constraint on the numerical/theorical exercises attempting to establish high-latitude equatorial electrojet coupling. 

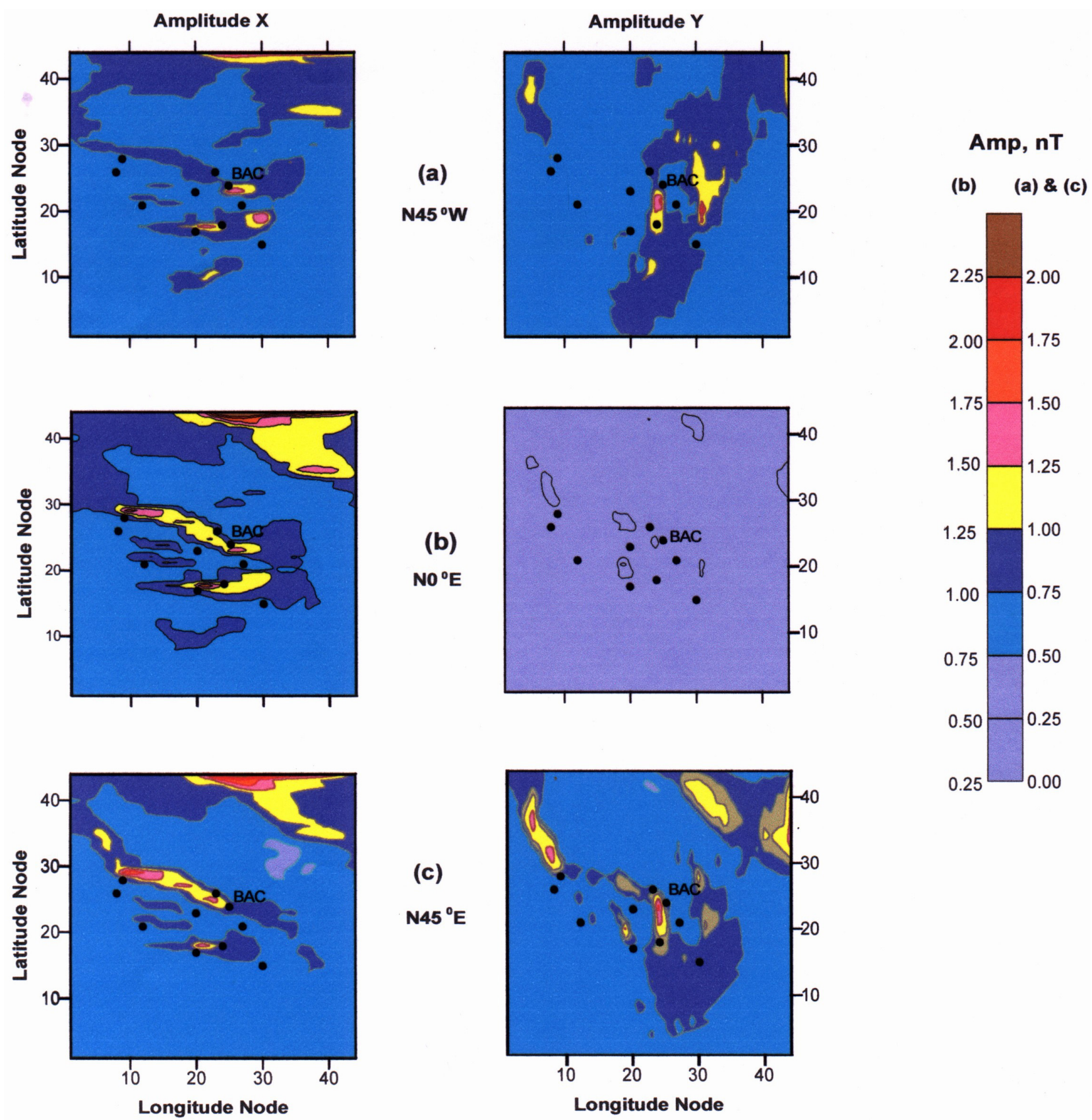

Fig. 6. Contour plots of $X$ and $Y$ amplitudes resulting from electromagnetic induction in laterally variable conductivity distribution map, shown in Fig. 2. Electromagnetic responses are computed for a oscillating source field of period 10 min and amplitude of $1 \mathrm{nT}$. Contour plots are shown separately when uniform source field is linearly polarized in (a) $\mathrm{N} 45^{\circ} \mathrm{W}$ (b) $\mathrm{N}-\mathrm{S}$ and (c) $\mathrm{N} 45^{\circ} \mathrm{E}$ directions.

\section{Estimation and elimination of the induction effects}

It is apparent that the true characterization of external source currents from ground-based magnetic observations requires a complete evaluation of the induction effects. This is difficult to achieve, as a unique solution to the induction problem would require exact knowledge of the source field, as well as the internal conductivity distribution, both radial and lateral. This becomes much more complicated in the equatorial or auroral electrojet regions where the fundamental assump- tion of spatially uniform source field, implicit in GDS and MT, is invalidated. How the non-uniformity of the source field modulates the induction process is yet not fully resolved (Mareschal, 1986; Pirjola, 1992; Chandrasekhar and Arora, 1994). However, if one can neglect the effect of non-uniformity on induction process, as the results of Padilha et al. (1997) warrant, a reasonable estimate of anomalous inductions, due to uniform source field, can be simulated. The maps of anomalous fields would serve to evaluate their importance and possibly correct the geomagnetic data for source 
(a) Source Field Polarization : N 45 degree W

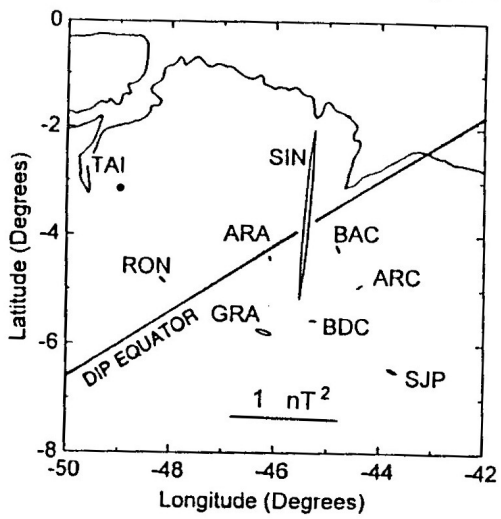

(b) Source Field Polarization : N 0 degree E

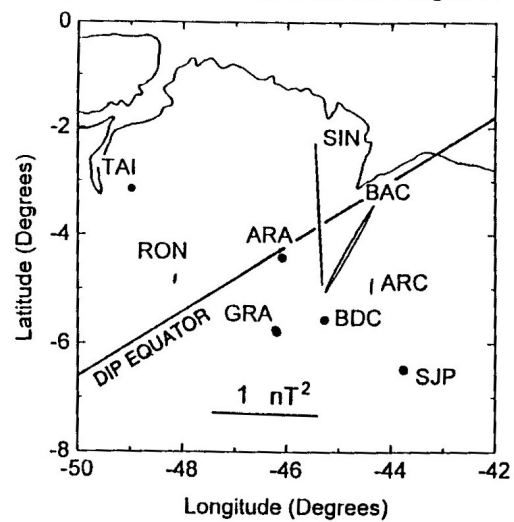

(c) Source Field Polarization : N 45 degree E

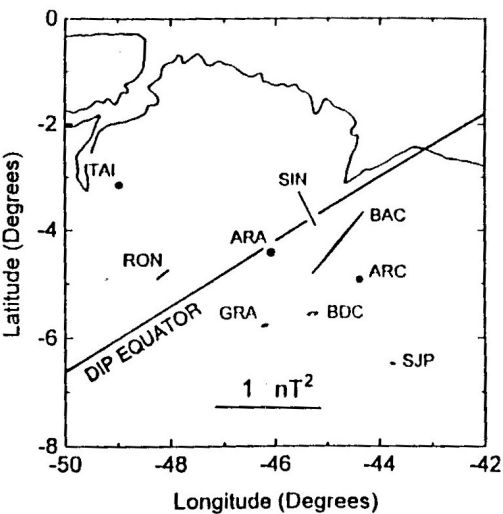

Fig. 7. Ellipses of anomalous horizontal field are shown for three polarizations of the source field.

field characterization. The two practical approaches to estimate the contribution of anomalous induction to horizontal field components are discussed below.

4.1 Numerical simulation of anomalous horizontal fields from conductance distribution map

In case the nature of electrical conductivity distribution in a given region is established from geomagnetic deep sounding or magnetotelluric techniques, the anomalous horizontal field that would be produced by an external field of given strength and direction (polarization) can be computed by recourse to numerical modelling. The simulated anomalous fields can then be used to correct the observed horizontal field components at individual sites for the anomalous induction effects. Figures 6 and 7 illustrate the application of this approach. As noted earlier, the nature of electrical conductivity distribution in the present study region has already been derived from the magnetometer array data (Fig. 2). The spatial variation of horizontal fields that would result from induction in this laterally variable conductance map by an external source field of given magnitude and polarization is calculated using thinsheet formulation of Vasseur and Weidelt (1977). In the numerical scheme adopted, the study area of $11^{\circ} \times 10^{\circ}$ (shown in Fig. 1) was divided into a $44 \times 44$ grid and the fields are computed at the center of each cell. It is known that induction in an elongated conductive structure maximizes when the source field is polarized at a right angle to the strike of the involved structure. Minimum response is observed in association with source field polarized parallel to the strike direction. In order to ascertain the extent of anomalous induction effects in differently oriented but mutually coupled structures, the induction response is worked for three different polarizations of source field, namely $\mathrm{N} 45^{\circ} \mathrm{W}, \mathrm{N}-\mathrm{S}$ and $\mathrm{N} 45^{\circ} \mathrm{E}$. In each case, the uniform source field had unit amplitude and induction responses were computed at the period of Pc5 pulsation, i.e. $10 \mathrm{~min}$. Figure 6 gives the spatial variation of total (normal + anomalous) fields in $X$ and $Y$ components for three assigned polarizations of the source field.
It is clearly noticed that horizontal fields are enhanced (as much as 40-50\%) right over the high conductivity zones, i.e. over the PBCA and LINK conductivity anomalies. However, the relative magnitudes and extent of anomalous fields in $X$ and $Y$ varies with the azimuth of the incident source field. NW polarized field produces strong anomalies both in $X$ and $Y$ in the region of PBCA, whereas NE polarization excites a strong anomalous response from the LINK anomaly. N-S polarization excites a response both from the PBCA and LINK anomalies, though only in the $X$ component.

It is also seen that large anomalous fields do not extend too far away from the high conductivity zones. As a working rule, stations located at distances more than 3-4 times the depth of the involved structure are not affected by the current concentration in the conductive body. The magnetic fields at such locations can then be defined as normal fields (external plus induction in layered regional structure). The normal fields, when subtracted from fields at all other nodes, provide an estimate of the anomalous horizontal field at the respective node. Adopting the computed horizontal fields at station GOI (see Fig. 1 for location) as a measure of normal fields, the anomalous fields in $X$ and $Y$ were obtained for nodes representing magnetometer sites. The amplitude and phase of anomalous horizontal fields in $X$ and $Y$ are then combined to construct ellipses of anomalous horizontal fields that are solely due to induction in structure, marked by large lateral conductivity contrast. The plots of anomalous ellipses associated with varying polarizations are shown in Fig. 7. Appreciably large ellipses at BAC and SIN bring out the anomalous character of horizontal fields resulting from induction at the junction of PBCA and LINK anomalies. However, the relative sizes and orientation of the anomalous fields varies with the polarization of the source fields. NW polarized source field produces anomalous horizontal fields only at station SIN. The magnitude of the anomalous field at SIN decreases as source polarization deviates from this angle. This reduction is rather sharp as source polarization swings from NS orientation to $\mathrm{NE}$ quadrant. In contrast, $\mathrm{BAC}$ registers strong anomalous fields when the source field is polarized along 
$\mathrm{N} 45^{\circ} \mathrm{E}$ and vanishes for orthogonal polarization. It was pointed out in Arora et al $(1998,1999)$ that concentration of induced currents in a narrow structure of large vertical extent beneath BAC accounts for the observed polarization dependences as well as for the anomalous large $Y$ fields at BAC. This geometrical concentration of the induced currents also leads to pinching (reduction) effect in the current distribution near the resistive side of the NW edge of the PBCA, accounting for the low values of $X$ and $Y$ at SIN. The relative amplitude and phase of the anomalous horizontal fields in relation to an external source field control the resultant polarization ellipse, as shown in Fig. 4. The horizontal field at GOI, as noted earlier, can be reckoned to be a measure of normal fields. For the pulsation event of 24 March 1991, the observed polarization ellipse at GOI is polarized along $\mathrm{N}$ $15^{\circ} \mathrm{E}$ and has an amplitude of $11 \mathrm{nT}$. When the anomalous fields corresponding to this magnitude and polarization are calculated, the amplitude and phase in $X$ and $Y$ components at BAC are in-phase with normal fields, but out-of-phase at SIN. As a consequence, they result in a sufficiently enlarged ellipse at BAC and an appreciably reduced ellipse at SIN, as observed in Fig. 7. In other words, the anomalous fields corresponding to the magnitude and polarization of the 24 March 1991 pulsations were estimated and eliminated from the observed fields at BAC and SIN; the resultant polarization ellipses, free from induction effect, become compatible with the adjoining stations and, thus, justify their interpretation in terms of the external sources.

\subsection{Simulation of anomalous horizontal fields through horizontal-field transfer function}

When the electrical conductivity distribution or data from a large array is not available, the alternative approach is to choose, on the basis of geological and geophysical information, a reference station. At this chosen site, the horizontal fields $\left(X_{n}, Y_{n}\right)$ are uncontaminated by lateral conductivity variation and, hence, can be considered as measure of normal fields. The Fourier transform of horizontal field components at a remote site $\left(X_{m}, Y_{m}\right)$ and a normal site $\left(X_{n}, Y_{n}\right)$ can then be related to establish inter-component frequency-dependent transfer functions (Schmucker, 1970), defined as follows:

$$
\begin{aligned}
X_{m} & =\left(1+T_{x x}\right) X_{n}+T_{x y} Y_{n}+\epsilon_{x} \\
Y_{m} & =T_{y x} X_{n}+\left(1+T_{y y}\right) Y_{n}+\epsilon_{y}
\end{aligned}
$$

The transfer functions are a set of $T$ 's, contain information on the anomalous conductive structure in the vicinity of a remote station. $\epsilon_{x}$ and $\epsilon_{z}$ are the error terms. In practice, a set of transfer functions are obtained by minimizing error terms in the least-square sense. Given estimates of transfer functions for the pulsation frequencies, the anomalous horizontal fields associated with an incident source field of given magnitude $(H)$ and azimuth $(\theta)$ can be obtained by performing hypothetical event analysis technique (Schmucker, 1970; Bailey et al., 1974) on these transfer functions

$X_{a}=T_{x x} H \cos \theta+T_{x y} H \sin \theta$,
$Y_{a}=T_{y x} H \cos \theta+T_{y y} H \sin \theta$.

The fields thus estimated can then be used to correct the observed fields for induction effects, prior to their application in the determination of polarization parameter or source field characterization.

\section{Concluding remarks}

The above field example clearly illustrates that if anomalous induction effects are not taken incognizant of the pulsation and other short period fluctuations studies, it can lead to erroneous characterization of the source field. Given that the subsurface conductivity distribution is more heterogeneous in the crustal section, the short period pulsations and storm sudden commencements, with appropriate frequency composition to penetrate these depths, are vulnerable to induction effects. The strength of anomalous induction effects in geomagnetic fluctuation is essentially a function frequency, controlled by the depth of electrical heterogeneities, but would also depend on the polarization of the source field with respect to the strike of the electrical discontinuity. The presence of anomalous induction effect can bias the estimate of equatorial enhancement, particularly if obtained from a single station. The reported longitudinal inequalities in equatorial enhancement of SSC and other short period may, in part, manifest from regional differences in induction effects, as hypothesized earlier by Singh et al. (1982). The presence of induction effects pose a difficulty in modeling the source currents system associated with polar magnetic substorms from ground-based magnetic observations as emphasized earlier by Mareschal and Kisabeth (1977) and Mareschal (1978).

Both in mircopulsation and GDS studies, deployments of magnetometer arrays are the common data collection tools. The $210^{\circ}$ E Meridian chain (Yumoto et al., 1995), IMS Scandinavian, European SAMNET, EISCAT and Australian magnetometer arrays are the classical examples that have been set up to investigate the ULF related magnetospheric process. Given that large-scale, 2-D magnetometer arrays have proved to be a useful and efficient method for delineating and mapping first-order conductivity structure, a number of nation-wide surveys have been undertaken to probe conductivity structures on continental/subcontinental scale. Australian Wide Arrays of Geomagnetic Stations (Chamalaun and Barton, 1993) and an array covering the whole of New Zealand (Chamalaun and McKnight, 1993) are some of the outstanding examples. Measurements of the time-varying geomagnetic components recorded successively at a series of stations across Japan (Fujiwara and Toh, 1996) and northern England (Banks et al., 1993) are also used to simulate anomalous induction effects at regional or sub-continental scale. The results from Japan convincingly show that strong regional anomalies associated with current channeling in sedimentary layers prevail in horizontal components (Fujiwara and Toh, 1996). The picture of internal conductivity distribution (Wang and Lilley, 1999) or geographic distribution of horizontal transfer functions can be used to estimate the 
extent and possibly to correct the induction effect in magnetic pulsation data. A better understanding of the internal and external contributions would permit a more precise interpretation of the observed geomagnetic variation in terms of magnetospheric process as well as deduction of the internal conductivity distribution. Some basic merits of integrating these studies are reviewed by Pilipenko and Fedorov (1994).

Acknowledgements. Authors wish to thank D. R. K. Rao for many fruitful discussions. Authors also wish to thank two external referees for their comments and suggestions on earlier version of the manuscript.

Topical Editor G. Chanteur thanks F. E. M. Lilley and another referee for their help in evaluating this paper.

\section{References}

Arora, B. R., Geomagnetic Deep Sounding, in: Natural source electromagnetic induction in the Earth. Eds. B. R. Arora and Sriniwas, New Age International Limited, Publishers, New Delhi, 80128, 1997.

Arora, B. R., Rigoti, A., Vitorello, I., Padilha, A. L., Trivedi, N. B., and Chamalaun, F. H., Electrical imaging of the intracratonic Parniaba basin. North-northeast Brazil, J. Geomagn. Geoelectr., 49, 1631-1648, 1997.

Arora, B. R., Rigoti, A., Vitorello, I., Padilha, A. L., Trivedi, N. B., and Chamalaun, F. H., Magnetometer array study in northnortheast Brazil: conductive image building and functional induction modes, Pure Appl. Geophys., 152, 349-375, 1998.

Arora, B. R., Padilha, A. L., Vitorello, I., Trivedi, N. B., Fontes, S. L., Rigoti, A., and Chamalaun, F. H., 2-D geoelectrical model for the Parnaiba Basin conductivity anomaly of northeast Brazil and tectonic implications, Tectonophysics, 302, 57-69, 1999.

Bailey, R. C., Edwards, R. N., Garland, G. D., and Greenhouse, J.P., Electrical conductivity studies over a tectonically active area in eastern Canada, J. Geomagn. Geoelectr., 26, 125-146, 1974.

Banks, R. J., Irving, A. A. K., and Livelybrooks, D. W., The simulation of magnetic variation anomalies using single-station data, Phys. Earth Planet. Inter., 81, 85-98, 1993.

Chamalaun, F. M. and Barton, C. E., The large scale electrical conductivity structure of Australia, J. Geomagn. Geoelectr., 45, 100 $121,1993$.

Chamalaun, F. M. and Maknight, J. D., A New Zealand wide magnetometer array study, J. Geomagn. Geoelectr., 45, 741-759, 1993.

Chadrasekhar, E. and Arora, B. R., On the source field geometry and geomagnetic induction in southern India, J. Geomagn. Geoelectr., 46, 815-825, 1994.

Egbert, G. D. and Booker, J. R., Imaging crustal structure in southwestern Washington with small magnetometer arrays, J. Geophys. Res., 98, 15967-15985, 1993.

Fujitani, S., Araki, T., Yumoto, K., Shiokawa, K., Tsunomura, S., Yamada, Y., Ichinose, T., Hayashi, K., Kitamura, T., Orr, D., Milling, D. K., Luhr, H., Rostoker, G., Rao, D. R. K., Singer, H., and Liu, C. F., Global Pc5 on March 24, 1991, STEP-GBRSCNEWS, Special Issue SOLTIP Data Analysis Activity, vol.4, no.1, 1994.

Fujiwara, S. and Hiroaki, T., Geomagnetic transfer functions in
Japan obtained by first order geomagnetic survey, J. Geomag. Geoelectr, 48, 1071-1101, 1996

Gough, D. I., and Ingham, M. R., Interpretation methods for magnetometer arrays, Rev. Geophys. Space Phys., 21, 805-827, 1983.

Hughes, W. J., Magnetospheric ULF waves: A tutorial with a historical perspective, in: Solar wind sources of magnetospheric ultralow-frequency waves, Geophysetson, K. Takahashi, M. Scholer, Geophysical Mon., 81, 1-12, 1994.

Kanasewich, E. R., Time sequence analysis in geophysics, pp. 328353, The University of Alberta Press, Alberta, 1981.

Mareschal, M., An estimate of the Earth's induction effects on the vertical polarization of the Pc5 pulsations recorded in auroral regions, Planet. Space Sci., 26, 1077-1080, 1978.

Mareschal, M., Modeling of natural sources of magnetospheric origin in the interpretation of regional induction studies: A review, Surv. Geophys., 8, 261, 1986.

Mareschal, M. and Kisabeth, J. L., Simulating the Earth's induction effects on substorm data recorded at mid-latitude stations: The three-dimensional problem, J. Geomag. Geoelectr., 29, 81-104, 1977.

Padilha, A. L., Vitorello, I., and Rijo, L., Effects of the equatorial electrojet on magnetotelluric surveys: Field results from northwest Brazil, Geophys. Res. Lett., 24, 89, 1997.

Pilipenko, V. A. and Fedorov, E. N., Magnetotelluric sounding of the crust and hydromagnetic monitoring of the magnetosphere with the use of ULF waves, in: Solar wind sources of magnetospheric ultra-low-frequency waves, Geophysetson, K. Takahashi, M. Scholer, Geophysical Mon., 81, 283-292, 1994.

Pirjola, R., On magnetotelluric source effects caused by an auroral electrojet system, Radio Sci., 27, 463, 1992.

Schmucker, U., Anomalies of geomagnetic variations in the southwestern United States, Bull. Scripps. Inst. Oceanogr., 13, 1-165, 1970.

Southwood, D. J., Some features of field line resonances in the magnetosphere, Planet. Space Sci., 22, 383-491, 1974.

Singh, B. P., Agarwal, A. K., and Carlo, L., Anomalies in $H$ at Indian equatorial stations and their effect on equatorial enhancements, J. Atmos. Terr. Phys., 44, 241-244, 1982.

Tsunomura, S. and Araki, T., Numerical analysis of equatorial enhancement of geomagnetic commencement, Planet. Space Sci., 32, 599-604, 1984.

Trivedi, N. B., Arora, B. R., Viera, L. E. A., Rao, D. R. K., and Yomoto, K., Ionospheric modulation of the Pc5 magnetic pulsations, 5th International Congress of the Brazilian Geophysicists, Rio de Janeiro, Sept. 28 - Oct. 2, 1997a.

Trivedi, N. B., Arora, B. R., Padilha, A. L., Rigoti, A., Da Costa, J. M., Dutra, S. L. G., and Chamalaun, F. H., Global Pc5 magnetic pulsations of March 24, 1991 as observed along the American sector, Geophys. Res. Lett., 24, 1683-1688, 1997 b.

Vasseur, G. and Weidelt, P., Bimodel electromagnetic induction in non-uniform thin sheets with an application to the northern Pyremean induction anomaly, Geophys. J. R. Astron. Soc., 51, 669690, 1977.

Wang, L.J. and Lilley, F. E. M., Inversion of magnetometer array data by thin-sheet modeling, Geophys. J. Int., 137, 128-138, 1999.

Yumoto, Y. and the $210^{\circ}$ MM Magnetic Observation Group, Initial results from the 210 Magnetic Meridian project - Review, J. Geomagn. Geoelctr. 47, 1197-1213, 1995. 